Background Use of SEBTm can identify athletes with risk of injury and help to set up prevention strategies.

Objective To identify subjects at risk of lower limb injuries (LLI) with SEBTm after a specific prevention training.

Design Cluster randomised controlled trial.

Setting French elite soccer youth academy.

Patients (or Participants) 36 healthy female athletes (aged 1519 years old) were included and assigned to the 'Control Groupe' (CrtGrp) or 'Prevention Training Group' (PrevGrp) by a cluster randomised controlled trial.

Interventions (or Assessment of Risk Factors) Both groups performed normal 5 weeks pre-season training, but in the intervention' we implemented a specific prevention protocol based on international recommendations.

Main Outcome Measurements Composite score (CS) of SEBTm were measured in both group at baseline (T0), after 5 weeks (post-protocol) and 3 months after the end of the protocol. Furthermore a 10 month follow up questionnaire post protocol was used to asses LLI in the two groups.

Results After 5 weeks and exclusion criteria, 28 players completed the all study (16 in the control group and 12 in the intervention group). After the protocol period, CS increased significantly in the PrevGrp $(p=0,03)$. In the CrtGrp, no difference was seen between the 2 testing sessions $(p=0,68)$. Based on injury risk factors, 19 subjects were identified as 'at risk' at T0. Of these, 4 players had at-least 1 LLI after 10 months $(R R=0,67)$. Moreover, 2 subjects who were not identified 'at risk' sustained a LLI during during the 10 months period. After 5 weeks, 2 of the 12 subjects in the PrevGrp exceeded the SC cut-off, compare to 0 on 16 players in the CrtGrp.

Conclusions This study suggests that the prevention training program seems to improve the SC at SEBT. This could leed to a diminished risk to sustain an LLI. These results needed to be confirmed with larger sample size studies.

\section{USING INFOGRAPHICS AND VIDEO TO PREVENT INJURIES AND ILLNESSES IN ATHLETICS}

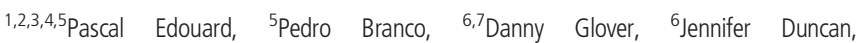
${ }^{8}$ Andy Richardson, ${ }^{5,9,10}$ Marianna Kiss, ${ }^{3,12}$ Frederic Depiesse, ${ }^{6,11}$ Andrew Murray. ${ }^{1}$ Interuniversity Laboratory of Human Movement Science (LIBM EA 7424), University of Lyon, University Jean Monnet, Saint-Etienne, France; ${ }^{2}$ Department of Clinical and Exercise Physiology, Sports Medicine Unit, University Hospital of Saint-Etienne, Faculty of Medicine, Saint-Etienne, France; ${ }^{3}$ Medical Commission, French Athletics Federation (FFA), Paris, France; ${ }^{4}$ Swiss Olympic Medical Centre, Centre de médecine du sport, Division de médecine physique et réadaptation, Centre Hospitalier Universitaire Vaudois, Lausanne, Switzerland; ${ }^{5}$ European Athletics Medical and Anti-Doping Commission, European Athletics Association (EAA), Lausanne, Switzerland; ${ }^{6}$ Knowledge Translation Team, Sport and Exercise, University of Edinburgh, Edinburg, UK; ${ }^{7}$ Health Education Yorkshire and the Humber, Leeds, UK; ${ }^{8}$ Institute of Cellular Medicine, Newcastle University, Newcastle Upon Tyne, UK; ${ }^{9}$ Hungarian Athletics Federation (Magyar Atlétikai Szovetség), Budapest, Hungary; ${ }^{10}$ National Institute for Sport Medicine, Budapest, Hungary; ${ }^{11}$ Human Performance Science Research Group, University of Edinburgh, Edinburg, UK; ${ }^{12}$ Department of Physical Medicine and Rehabilitation, University Hospital of Martinique, Le Lamentin, Martinique, France

\subsection{6/bjsports-2021-IOC.256}

Background Injuries and illnesses represent a health problem affecting athletics athletes' training and performance. Recently, research on injury and illness epidemiology and risk factors have provided some insights to help implement prevention strategies. The next step is presenting the science, in an engaging form that can assist medical teams, coaches and athletes understand and implement these strategies.
Objectives To produce resources in order to disseminate knowledge on injuries and illnesses epidemiology and risk factors, and highlight potential prevention strategies.

Participants Researchers and practitioners working in athletics injury and illness prevention collaborated with athletes.

Main Outcome Measurements Resources/communication assets, for example infographics, animations, video, were produced and shared with medical teams, athletes and coaches.

Results Infographics, animation and engaging knowledge translation tools/educational materials on illness epidemiology during international athletics championships were produced. These took the scientific knowledge, made it specific for the context of athletics, and provided easily digestible, visually rich information. These were shared with athletes at events such as the European Athletics Indoor Championships.

Conclusions We developed resources to be shared to all stakeholders working with athletics athletes, providing tips and practical recommendations on injury and illness prevention. Having share these to athletes and their teams, the next step is to evaluate the documents and tools for and by athletes and involved actors, and the effectiveness of these interventions. These strategies may be relevant to provide athletes with engaging information on preventing injury and illness in other sports.

\section{FIELD HOCKEY INJURIES AND PERSONAL PROTECTIVE EQUIPMENT: A STATUS SURVEY OF GERMAN NATIONAL TEAMS}

Sabrina Gorks, Thomas Henke, Petra Platen. Ruhr-University Bochum, Dep. of Sports Medicine, Bochum, Germany

\subsection{6/bjsports-2021-IOC.257}

Background In order to prevent sports injuries, it is necessary to know the number of injuries and injury mechanisms of sports-related injuries. In addition, acceptance and use of personal protective equipment (PPE) should be examined. The results give a better overview in German elite hockey.

Objective Data on injury frequencies and injury patterns in field hockey as well as on the wearing behavior of PPE in training and competition.

Design Questionnaire-based retrospective total survey. Players were instructed to fill out one general and, in case of at least one time loss injury (at least 1 training or competition missed) during a 3-year-period (February 2016 - April 2019), one additional questionnaire per injury.

Setting National elite hockey level. Participants were member of the German national teams including A-, U 21- and U 18men and women teams (age range 16-32y).

Participants 127 players took part in the survey.

Main Outcome Measurements Exposure, injured body parts, incidence, time loss, wearing of PPE

Results 89 players sustained 114 injuries. Incidence: Competition: Men: 5.79 injuries per 1000h; Women: 7.99 injuries per 1000h. Training: Men: 0.51 injuries per 1000h; Women: 0.31 injuries per 1000h. Body parts: Men: 77.6\% lower extremities, 8.95\% upper extremities. Women: 75\% lower extremities, $16.7 \%$ upper extremities. Severity defined by Fuller et al. (2006): Men: 53.3\% severe, 30\% moderate, $10 \%$ mild and $6.67 \%$ minimal. Women: $47.8 \%$ severe, 37\% moderate, $13 \%$ mild and $2.17 \%$ minimal. PPE: Fielders: Men: leg protection 96.6\%, mouthguard $88.1 \%$ and gloves $66.1 \%$. Women: leg protection 98\%, mouthguard $94 \%$ and gloves 94\%. Goalkeeper: carry more PPE in addition to mandatory PPE. 
Conclusions Injury incidence in German elite hockey players were lower than in comparable studies. Localization was almost similar to findings in other studies. No/less other studies recording the wearing behavior of PPE. Injury mechanism and correlation with wearing behavior of PPE should be examined to develop further preventive measures.

\section{\begin{tabular}{|l|l}
\hline 280 & IMPLEMENTATION OF INJURY PREVENTION PROGRAMS
\end{tabular} AFTER THEIR INITIAL EFFECTIVENESS TRIAL}

'Jelena Haugg, ${ }^{1}$ Evert Verhagen, ${ }^{1}$ Joske Nauta, ${ }^{1}$ Ingrid Vriend, ${ }^{2}$ Carly D McKay, ${ }^{1}$ Caroline Bolling, ${ }^{1}$ Femke van Nassau. ${ }^{1}$ Amsterdam Collaboration on Health and Safety in Sports, Department of Public and Occupational Health, Amsterdam UMC, Vrije Universiteit Amsterdam, Amsterdam Movement Sciences, Amsterdam, Netherlands; ${ }^{2}$ Centre for Motivation and Health Behaviour Change, Department for Health, University of Bath, Bath, UK

\section{$10.1136 /$ bjsports-2021-IOC.258}

Background There is a gap between evaluating injury prevention interventions and use in practice. Implementation research aims to transfer interventions beyond research.

Objective The aim of this study was to find out whether what implementation processes took place during effectiveness studies targeting injury prevention programs, what factors hindered or facilitated this process and what activities and support were provided to promote implementation during the trial.

Design We conducted a systematic review and sent out an online survey targeting authors of included studies.

Setting Papers investigating all levels of sports (amateur, general sporting population, recreational, university, youth, and professional) were included.

Patients (or Participants) Authors of studies included in the review reporting effectiveness evaluations of injury prevention programs were contacted. Papers eligible for the survey were depicted from the review from Vriend et al. (2017) $(\mathrm{n}=81)$ and updated papers $(\mathrm{n}=31)$, which resulted in 112 included papers, describing 105 injury prevention programs.

Interventions (or Assessment of Risk Factors) The survey took 15 minutes to complete and could be taken only once. Participants were contacted by email and received a reminder twice. Main Outcome Measurements The survey asked what materials or activities were used during the trial to improve the delivery, use in practice, compliance and/or adherence, monitoring of this process (i.e. compliance, adherence, exposure) and what hindered or facilitated this implementation process.

Results In total, 33 participants completed the survey. Personal face to face education and the providence of supportive material were the most used materials and activities for implementation. Compliance to the program was a key factor for implementation, and could be both, facilitator or barrier.

Conclusions This study provides initial insight into implementation activities and processes conducted during a trial. To ensure a greater impact, future studies should document their activities more detailed and formally.

\section{KINESIOTAPING; DOES IT REALLY PREVENT SPORTS INJURIES?}

Yonatan Kaplan. Jerusalem Sports Medicine Institute, Jerusalem, Israel

$10.1136 /$ bjsports-2021-IOC.259

Background Over the last 50 years kinesiotaping has become a very popular adjunct in the treatment of sports injuries. Its role in sports injury prevention however remains unclear.

Objectives To investigate whether kinesiotaping has a role in the prevention of sports injuries.

Methods An electronic search was conducted up to June 2019 using medical subheadings and free-text words. The subjectspecific search included the terms 'Kinesio tape', 'injury prevention', 'motor control', and 'proprioception'. The search included levels 1, 2 and 3 evidence-based articles.

Results Twenty-one articles were found. The majority of the articles did not support the use of Kinesio tape in the prevention of injury nor in the increase of joint positioning sense, proprioception or increased motor control.

Conclusions It remains questionable whetherKinesio tape should be used in order to prevent sport injury.

\section{A 'STEP' TOWARDS PREVENTION OF OA IN PHYSICALLY ACTIVE AMPUTEES USING A NOVEL FOOT PROSTHESIS: A RANDOMISED CONTROLLED CROSS-OVER TRIAL}

1,4Phoebe Runciman, ${ }^{2,3}$ John Cockcroft, ${ }^{3,4}$ Wayne Derman. 'Department of Sport Science, Stellenbosch University, Cape Town, South Africa; ${ }^{2}$ Central Analytic Facility, Stellenbosch University, Cape Town, South Africa; ${ }^{3}$ Institute of Sport and Exercise Medicine, Stellenbosch University, Cape Town, South Africa; ${ }^{4}$ IOC Research Center, Pretoria, South Africa

\subsection{6/bjsports-2021-IOC.260}

Background Individuals with unilateral transtibial amputation (UTTA) have higher external ground reaction forces in joints on their sound side during gait and this been linked to increased risk of osteoarthritis in these individuals.

Objective To describe the biomechanical characteristics in UTTA whilst using a novel, mechanically powered energy storing and returning (ESAR) ankle/foot prosthesis, compared with two control foot prostheses.

Design Randomised cross-over trial.

Setting Laboratory based study.

Participants 20 participants performed a standardized selfpaced walking gait protocol using three different ankle/foot prostheses (novel ESAR foot (NOVEL), current gold standard ESAR (ESAR) foot and solid-ankle cushioned-heel (SACH) foot), in a randomised cross-over design.

Assessment of Risk Factors Three-dimensional kinematics of the lower body as well as ground reaction forces (GRF) were recorded with a ten-camera stereophotogrammetry motion capture system and three floor-imbedded force plates.

Main Outcome Measurements GRFs, external adduction moments (EAM) \& ankle range of motion (ROM)

Results Despite similar peak GRFs between feet on the prosthetic side during walking ( $\pm 108 \%$ body weight), there were higher GRFs on the sound side during the load acceptance phase whilst participants used the SACH $(126.3 \pm 11.5 \%)$ and ESAR $(116.8 \pm 10.5 \%)$ feet, but not the NOVEL (108.7 \pm 9.3\%, $\mathrm{p}<0.001)$. External Abduction Moments (EAM) observed on the sound side during loading was $0.55 \pm 0.3 \mathrm{Nm} / \mathrm{kg}, 0.6$ $\pm 0.3 \mathrm{Nm} / \mathrm{kg}$ and $0.7 \pm 0,31 \mathrm{Nm} / \mathrm{kg}$ for the NOVEL, ESAR and SACH. The NOVEL had a sagittal ROM of $21.4 \pm 3.2^{\circ}$, the ESAR $18.2 \pm 3.3^{\circ}$ and the SACH $8.4 \pm 2.3^{\circ}(\mathrm{p}<0.001)$.

Conclusions The use of the NOVEL foot resulted in a similar loading/force pattern between prosthetic and sound sides in UTTA, which was not seen with the two other foot prostheses. Furthermore, participants demonstrated a better transition during double support, with improved ROM, and EAM, whilst using the NOVEL. 